\title{
Problems Found Using a Radon Stripping Algorithm for Retrospective Assessment of Air Filter Samples
}

\author{
----- Operational Topic -----
}

\author{
Robert B. Hayes \\ National Security Technologies, LLC \\ P.O. Box 98521, MS RSL-47 \\ Las Vegas, NV 89193 \\ (702) $794-8825$ \\ hayesrb@nv.doe.gov
}

Keywords: air monitoring, radon, algorithm, PIPS, transuranic, TRU, alpha particles, beta particles. 


\begin{abstract}
An evaluation of a large number of air sample filters was undertaken using a commercial alpha and beta spectroscopy system employing a passive implanted planar silicon (PIPS) detector. Samples were only measured after air flow through the filters had ceased. Use of a commercial radon stripping algorithm was implemented to discriminate anthropogenic alpha activity on the filters from the radon progeny. When uncontaminated air filters were evaluated, the results showed that there was a timedependent bias in both average estimates and measurement dispersion of anthropogenic activity estimates with the relative bias being small compared to the dispersion, indicating that the system would not give false positive indications for an appropriately set decision level. By also measuring environmental air sample filters simultaneously with electroplated alpha filters, use of the radon stripping algorithm demonstrated a number of substantial unexpected deviations from calibrated values indicating that the system would give false negative indications. Use of the current algorithm is, therefore, not recommended for general assay applications. Use of the PIPS detector should only be utilized for gross counting without appropriate modifications to the curve-fitting algorithm. As a screening method, the radon stripping algorithm might be expected to see elevated alpha activities on air sample filters (not due to radon progeny) around the 200 disintegrations per minute level.
\end{abstract}




\section{Introduction}

A number of technologies have been realized for rapid retrospective assessment of anthropogenic activity on air samples. These include liquid scintillation (Metzger et al. 1995), Frisch grid alpha spectrometry (Scarpitta et al. 2000), use of a commercial continuous air monitor (CAM) with flow stopped (Hayes et al. 2005) or curve fitting decay curves (Hayes and Chiou 2003). Typically, these technologies would be used for a rapid assessment of an air sample in the vicinity of a nuclear facility or event where a CAM is not available or practical, as is the case for simple environmental air samples, routine air monitoring samples, or when an unplanned radiological release is suspected or known, resulting in emergency response-type applications where large numbers of high volume air samples will need to be screened and assayed as rapidly as possible.

\section{Experiment}

This study started using 42 air samples taken over a period of a few months. These air samples were known not to contain any contamination above that already present in the environment around the Waste Isolation Pilot Plant (WIPP) (Arimoto et al. 2002) due to atmospheric testing of nuclear weapons. The bias and dispersion in these uncontaminated filters was then evaluated utilizing data taken more than 1,000 minutes post sampling. Final validation attempts of the method utilized electroplated sources placed behind additional air sample filters after an appropriate radiation shield (with a matching hole in it) was placed over the source but under the air sample. This was done previously by Hayes, et al. (2005) after the air sample filter had a concomitant hole punched in it (see Figure 1). In this way, the radon stripping algorithm could be tested with anthropogenic alpha activity present in tandem with the full radon progeny distribution.

The present study was carried out using the Canberra ${ }^{\dagger}$ iSolo $^{\circledR}$ counter, which uses a passive implanted planar silicon (PIPS) detector and a custom algorithm (Canberra 2004). Measurements of effluent air samples were taken at the WIPP using Canberra's custom system (Jierree et al. 2002). Roughly half the air samples taken were of actual ventilation effluent coming from the underground repository. The other samples were taken from a

\footnotetext{
${ }^{\dagger}$ Canberra Industries, Inc., 800 Research Parkway, Meriden, Connecticut 06450.
} 
sheltered outdoor location using a portable air sampler. Typical air flow rates were 2 cubic feet per minute (cfm) through $47 \mathrm{~mm}$ diameter air filters. As a general rule, all the effluent air samples would contain varying amounts of salt dust $(\mathrm{NaCl})$, whereas the portable air samplers would contain common surface particulate (akin to that measured by Arimoto et. al. 2002).

All air samples utilized a minimum of three hours of sampling time prior to measurement, but many took samples for 24 hours, or more. The measurements were typically multiple sequential 5-minute counts so as to trend all time dependencies present for this assay technique.

Each day, the experiment would start with a 30-minute background count to correct all subsequent measurements for that day. All of the subsequent net counts generated that day were then printed out and manually entered into a database for analysis. The alpha measurements would come from the 3 to $5.5 \mathrm{MeV}$ energy range of elevated counts. Air samples taken from previous days would often be measured on subsequent days, as well as samples taken that same day, so that trends could be evaluated over long time periods. Typically, samples would be measured using the same five-minute repeating count sequence for 24 iterations. Typically, one sample would be set at the end of the day to count a similar five-minute sequence, but for 100 iterations (overnight). Alpha efficiency was measured using plutonium-239 $\left({ }^{239} \mathrm{Pu}\right)$ sources. These measurements resulted in an overall efficiency value of $33.48 \%$.

The configuration shown in Figure 1 required a recalibration of the sources because the activity seen by the detector had to be determined for each source (as the uniformity of the activity on the sources was not assured). Furthermore, the shielding used for the sources was not perfectly centered, so multiple measurements were made of each source with its shield using iterative $90^{\circ}$ turns between each measurement. In this way, the expected values and their dispersion could be estimated and later compared with the values calculated from actual used air filters where the radon stripping algorithm would be implemented. The shielding used was composed of three pieces of thin waxed paper. This was empirically found to give zero alpha counts when they were placed over an electroplated source with no central hole present in the paper. This process was carried out using both alpha and beta sources, although the beta sources had to use much thicker 
shielding. The results of the beta measurements (taken in the configuration of Figure 1) are not reported here because the thickness of the required shield placed the air filters so close to the detector that the vendor later noted that the algorithm would be compromised because of the substantial reduction in air attenuation from the sample to the detector.

Air samples measured simultaneously with partially shielded sources were only measured for the first few hours post flow cessation. The study began in April 2005 and continued until July 2005.

\section{Results}

\section{Air Samples Alone}

The average air sample activity values are shown in Figures $2 \mathrm{a}$ and $2 \mathrm{~b}$ for up to 1,000 minutes post flow cessation (data taken beyond 1,000 minutes did not show any temporal dependence of interest). Also, the anthropogenic alpha and beta estimates from these environmental samples are shown in Figure 2a and Figure 2b, respectively. Along with the average values for each time interval, the associated one standard-deviation dispersion is shown measured as error bars. Here it can be seen that there is a timedependent bias for the first 200 minutes. Similarly, there is also a time-dependent dispersion about the measurements. The overall trends for the alpha and beta are qualitatively similar ; the primary difference being that the bias and dispersion in the beta measurements are almost five times that found with the alpha results.

After approximately 200 minutes, the bias and dispersion are minimized suggesting this time as an optimal delay post-flow cessation for counting the uncontaminated air filters. The dispersion continues to decrease over time, although this is not shown in these figures. After the 15 minute mark, the $t$-statistic for all of the values up to 200 minutes post-flow cessation is negative. By conducting a $t$-test on each five minute increment from all the measurements up to 200 minutes, 23 of the 40 values failed to have their mean be found statistically indistinguishable from zero at the $95 \%$ confidence level. A check was made to determine if there was any statistically significant difference between the results found from the effluent air filters and those from the portable air samplers. A difference of means hypothesis test on the data given in Table 1 shows that 
there is no statistically significant difference in these two groups for either the alpha or beta results. This is true for both before and after the 200-minute mark.

\section{Air Samples in Tandem with Electroplated Alpha Sources}

Using the configuration shown in Figure 1, multiple measurements were typically made up to about two hours after the flow had stopped. The results from using ${ }^{239} \mathrm{Pu}$ sources are shown in Figure 3. It can be seen that the transuranic (TRU) activity estimated, using the radon stripping algorithm, systematically underestimates the expected activity values taken during the calibration of the partially-shielded sources. The abscissa points show the average calibrated activity using only the shielded sources, along with the dispersion in these values, as horizontal error bars at the one standard deviation level. The ordinate values are the average from an approximately two-hour sequence of multiple five-minute counts post flow cessation of the used filters in the configuration shown in Figure 1. The vertical error bars are again at the one standard deviation level and represent the dispersion in each of the sequential five-minute counts. A weighted least-squares fit to the data in Figure 3 was done using the Levenberg-Marquardt algorithm where the individual weights were the quadrature sum of the abscissa and ordinate error values. This resulted in a fitted function of $Y=m X+b$, where $m=0.81 \pm 0.03$ and $b=-17 \pm 5$ dpm. The correlation coefficient was 0.966, although the chi-squared value was 41 , indicating that the individual errors did not reflect the actual dispersion of the data around the fit. Note that for the 16 fitted points (14 degrees of freedom), the $99.5 \%$ upper confidence limit on the chi-squared distribution would only be 31 .

The calculated beta activities using the ${ }^{239} \mathrm{Pu}$ sources were found to be correlated with the calculated alpha activities. The data given in Table 2 shows the calculated alpha and beta activities from each of the ${ }^{239} \mathrm{Pu}$ measurements, along with the dispersion in the series of counts. Another least-squares fit, using the alpha and beta errors summed in quadrature for weights, resulted in a fit result to $\mathrm{Y}=\mathrm{m} \cdot \mathrm{X}+\mathrm{b}$ of $\mathrm{m}=0.503 \pm 0.070$ and $\mathrm{b}=0 \pm 9 \mathrm{dpm}$ with the correlation coefficient being 0.7 (here $\mathrm{X}$ is the alpha activity and $\mathrm{Y}$ is the beta activity). The chi-squared value was 58 indicating again that the measured errors used did not represent the data distribution in the functional fit. Some time dependence was also seen for the beta predictions but not on all samples. When this was seen, the effect was a 
trend toward zero in all cases, although the measurements did not extend beyond 200 minutes in these cases.

Two americium-241 ( $\left.{ }^{241} \mathrm{Am}\right)$ sources were also evaluated in the same fashion as the multiple ${ }^{239} \mathrm{Pu}$ sources. These were of a much higher activity (shielded activity values of approximately $2 \times 10^{3}$ and $6 \times 10^{4} \mathrm{dpm}$ ) with both showing a calculated value of approximately $45 \%$ of the calibrated values (note that the ${ }^{239} \mathrm{Pu}$ sources averaged about 78\%). Although these two data points are not consistent with the linear fit shown in Figure 3, they do indicate an underestimate of the calculated TRU activities using the radon-stripping algorithm. Likewise, the beta activity estimated for the lower and higher activity ${ }^{241}$ Am sources were $-15 \pm 65 \mathrm{dpm}$ and 2,564 $\pm 68 \mathrm{dpm}$, respectively (the latter value could be due to the Photoelectric effect betas coming from the $59 \mathrm{keV}$ gamma ray emission from the $\left.{ }^{241} \mathrm{Am}\right)$. The gamma interaction may be related to the difference in the americium and plutonium source measurements.

The results of the linear fit to the ${ }^{239} \mathrm{Pu}$ sources in Figure 3 would suggest a $19 \%$ underestimate of the alpha activity with a negative $16 \mathrm{dpm}$ bias. The empirical deviation found by taking the expected values, minus the calculated values, was $-74 \pm 63 \mathrm{dpm}$. A decision level could be formed from this result at the 95\% confidence level if the actual TRU activity were $74+1.645 * 63 \mathrm{dpm}=178 \mathrm{dpm}$ or above, then the algorithm could be expected to give a positive alpha estimate indicating the presence of TRU activity. This assumes the bias effect is constant over the range (i.e., a slope of unity in Figure 3). Given the large deviation seen with the ${ }^{241} \mathrm{Am}$ results (45\%) relative to that estimated from the ${ }^{239} \mathrm{Pu}$ results from a linear fit (19\%), a quantitative decision limit does not seem reasonable, although rounding up to $200 \mathrm{dpm}$ for the moment seems a reasonably conservative estimate based on the data at this time.

\section{Using Vendor Recommended Count Procedures.}

After this study had been completed and found to give false negative results, the vendor made the recommendations that count times be set to 10 - 20 minutes and that a wait time of at least 15 minutes be utilized, with a preferable wait time of 30 - 40 minutes to obtain optimal results. Although the samples cannot be recounted, the results can easily be evaluated when only considering counts after 15 - 40 minutes of wait time have occurred, and sequential five-minute values can be averaged giving an approximate of the 10 to 20 
minute count result that would have otherwise been obtained. This latter calculation would not account for any additional scatter caused by use of the default algorithm when only evaluating 5 minute spectra as opposed to 20 minute spectra, if specific bias were to occur outside simple statistical counting fluctuations. The resultant count results are shown in Figure 4 for the TRU activity data.

\section{Discussion}

The results of Figures $2 \mathrm{a}$ and $2 \mathrm{~b}$ show that due to the dispersion in the measurements anthropogenic activity estimations from environmental air samples post-flow cessation are not distinguishable from zero. If measurements are taken after the initial 200-minute window post-flow cessation, no bias is expected in environmental samples at the precision measured in this study and measurement dispersion should be at a minimum. As such, one might have considered two decision levels, the first would be for measurements made within the first 200 minutes of flow cessation and the second would be anytime after this. A more accurate analysis would ascribe a time dependency to these decision levels due to the effects seen in Figures 2A and 2B.

Given the large bias and perturbation on the alpha results shown in Figure 3, when artificial sources are counted simultaneously with the environmental air sample filters decision levels should be based on the latter if these could be reliably quantified. Given the unpredictable nature of the algorithm results when calculating alpha and beta activities on air filters after using the radon stripping algorithm, it is not recommended that the results obtained from this method be considered sufficiently reproducible to give a definitive number for the decision limits. As a first approximation, the results presented here indicate that the algorithm will correctly identify the presence of alpha or beta activity not arising from the radon progeny only if the former is on the order of a few

hundred dpm. In principle, an alternate approach, or algorithm, should be implemented if radon discrimination is to be employed such as that tested and validated by Hayes et al. (2005). No measurements were made with mixed alpha and beta electroplated sources, so the issue of mixed sources cannot be definitively addressed.

The results in this study may be attributed to the use of the radon stripping algorithm to remove counts due to radon progeny. In principle, this would be expected to be a fully valid and appropriate method; although, in this case, the algorithm is the same as that 
used in continuous air monitors (CAMs) and was initially developed for CAMs. As such, a CAM is continually placing more activity on the filter with time such that after 30 to 60 minutes, the amount of activity being deposited per unit of time will be equivalent to the activity decaying per unit of time. Now, although these algorithms should handle the temporal disequilibria that comes with diurnal increases and decreases in the parent radon levels (NCRP 1998), this is not the same dynamics one would expect from completely stopping the air flow and removing any new parent source terms of activity on the filter with time. As has been shown elsewhere (Hayes 2003) for continual flow conditions with disequilibria, less dominant decay emissions can cause undesirable bias in algorithm calculations if not accounted for during changes in the parent radon concentrations. In the present case, dominant contributions from short lived radon progeny (e.g., ${ }^{214}$ Po and ${ }^{218} \mathrm{Po}$ ) will very quickly cease to make dominant spectral contributions which will allow bias from Thoron $\left({ }^{220} \mathrm{Rn}\right)$ and Actinon $\left({ }^{219} \mathrm{Rn}\right)$ sources to potentially become non-negligible.

Another point raised by the vendor after the completion of this study was that the configuration in Figure 1 is not the designed configuration for using this instrument (having multiple sources in multiple planes). As with any air filter counting instrument, all sources are intended to be on the same plane as the air filter having the same sourceto-detector distance. Although the latter was a result of the desire to not have contaminated air filters, any spectral distortion would occur in the direction of higher energy peaks in the radon spectrum due to less air attenuation. Because these were environmental air samples, sample self attenuation would play a competing effect of smearing the spectra giving lower energy alpha peak locations. The extent to which this was a factor in this study cannot be determined based on the results obtained, but it was not believed to have affected the conclusions. 


\section{Conclusion}

This study evaluated the use of a PIPS detector with a radon stripping algorithm as a viable method for screening air sample filters' post-flow cessation. Time-dependent bias, along with time-dependent dispersion, was discovered on uncontaminated filters, although the bias was small relative to the dispersion.

When superimposed source activity was present along with the radon progeny on the filter, a substantial bias was seen with an underestimate taking place on the TRU activity. This was found to also result in an overestimate of the beta activity when only superimposed alpha activity was present.

This study shows the need for only employing technology that has been fully verified and validated through rigorous testing protocols prior to use in nuclear safety applications; use of the evaluated technology is recommended solely for gross counting purposes or validated algorithms, if available.

\section{Acknowledgements}

This manuscript has been authored by National Security Technologies, LLC, under Contract No. DE-AC52-06NA25946 with the U.S. Department of Energy. The United States Government retains a non-exclusive, paid-up, irrevocable, world-wide license to publish or reproduce the published form of this manuscript, or allow others to do so for United States Government purposes.

The measurements and data collection portion of this work was done by Washington TRU Solutions, LLC, under Contract No. DE-AC04-01AL66444 with the U.S. Department of Energy.

Special thanks are also extended to Don Harward and Sandra Gogol for enabling this work to be done. 


\section{References}

Arimoto R, Kirchner T, Webb J, Conley M, Stewart B, Schoep D, Walthall M. “239,230Pu and inorganic substances in aerosols form the vicinity of a waste isolation pilot plant: the importance of resuspension. Health Physics 83:456-470; 2002.

Canberra, iSolo ${ }^{\circledR}$ Alpha/Beta Counting System Algorithm Manual. Canberra Industries, Meriden, CT. 2004.

Hayes RB. "False CAM alarms from radon fluctuations.” Health Physics. 85: S81-S84; 2003.

Hayes RB, Chiou HC. "Curve fitting air sample filter decay curves to estimate transuranic content.” Health Physics 86: 80-91; 2003.

Hayes RB, Pena AM, Goff TE. "Use of alpha spectroscopy for conducting rapid surveys of transuranic activity on air sample filters and smears.” Health Physics 89: 172-18;: 2005.

Jierree C, Frank-Supka L, Waymire G, Hayes R. "Effluent and environmental monitoring challenges for an underground transuranic mixed waste disposal site.” Spectrum 2002, $9^{\text {th }}$ Biennial International Conference on Nuclear and Hazardous Waste Management, August 4-8, Reno, NV, American Nuclear Society, CD-ROM; 2002.

Metzger RL, Jessop BH, McDowell BL. “Bioanalysis of Uranium, Plutonium, and Curium on breathing zone air sample by solvent extraction and PERALS spectroscopy.” Radioactivity and Radiochemistry 6(3);46-55: 1995.

National Council on Radiation Protection and Measurements. "Exposure of the Population in the United States and Canada from Natural Background Radiation.” NCRP Report No. 94. Bethesda, MD; 1998.

Scarpitta S, Carte N. "Rapid Analytical Technique to Identify Alpha Emitting Isotopes in Water, Air Filters and Solid Matrices.” BAER 2000, 46 ${ }^{\text {th }}$ Annual Conference on Bioassay, Analytical, and Environmental Radiochemistry. Seattle, WA, November 12-17, 2000.

Salvatore SC, Miltenberger RP, Carte N. "Use of a Frisch grid ionization chamber to rapidly characterized and quantify a-emitting actinites in unprocessed soils, liquids, solids and air-dust samples.” BAER 2000, $46^{\text {th }}$ Annual Conference on Bioassay, Analytical, and Environmental Radiochemistry, Seattle, WA; 12-17 November 2000. 


\section{Tables}

Table 1. Grouped statistical measures from the environmental air sample filters measured alone. All values are given in dpm. The sigma values are at the one standard deviation level.

\begin{tabular}{lrrrr}
\hline & $\begin{array}{l}<200 \\
\text { minutes } \\
\text { mean }\end{array}$ & $\begin{array}{l}<200 \\
\text { minutes } \\
\text { sigma }\end{array}$ & $\begin{array}{l}>200 \\
\text { minutes } \\
\text { mean }\end{array}$ & $\begin{array}{l}>200 \\
\text { minutes } \\
\text { sigma }\end{array}$ \\
\hline All alpha measurements & -49.97 & 149.94 & -5.48 & 58.85 \\
Station A alpha values & -158.68 & 125.04 & -26.20 & 46.75 \\
Portable alpha values & 6.16 & 129.56 & 9.21 & 62.05 \\
All beta measurements & -9.39 & 22.53 & 0.86 & 4.94 \\
Station A beta values & -15.81 & 26.66 & 0.29 & 5.14 \\
Portable beta values & -6.07 & 19.26 & 1.25 & 4.76 \\
\hline
\end{tabular}


Table 2. Reconstructed activities using the experimental setup shown in Figure 1 and various ${ }^{239} \mathrm{Pu}$ sources. All values are given in dpm with the reported errors all being listed at the one standard deviation level.

\begin{tabular}{rrrr}
\hline $\begin{array}{l}\text { Alpha } \\
\text { activity }\end{array}$ & \multicolumn{1}{l}{$\begin{array}{l}\text { Alpha } \\
\text { error }\end{array}$} & \multicolumn{1}{l}{$\begin{array}{l}\text { Beta } \\
\text { activity }\end{array}$} & \multicolumn{1}{l}{$\begin{array}{l}\text { Beta } \\
\text { error }\end{array}$} \\
\hline-39.1 & 21.0 & -21.2 & 38.2 \\
-19.8 & 16.5 & -23.1 & 23.9 \\
38.2 & 12.5 & -14.1 & 2.4 \\
8.7 & 18.0 & 152.0 & 53.5 \\
-23.0 & 22.2 & 7.5 & 33.3 \\
102.7 & 36.4 & -86.8 & 58.6 \\
-30.2 & 19.9 & -39.3 & 24.3 \\
219.3 & 11.5 & 70.0 & 32.9 \\
7.4 & 6.7 & 62.6 & 22.1 \\
236.3 & 18.7 & 212.7 & 44.3 \\
-77.2 & 48.7 & -139.3 & 45.1 \\
326.3 & 38.3 & 124.3 & 102.0 \\
1.0 & 11.6 & 185.5 & 38.8 \\
492.0 & 30.0 & 454.6 & 98.0 \\
668.6 & 24.9 & 316.1 & 53.1 \\
\hline
\end{tabular}




\section{FigureCaptions}

Figure 1. Experimental configuration used for attempted verification and validation of technique. Drawing is qualitative only and not scaled in any direction.

Figure 2a. Estimated anthropogenic alpha and beta activity on environmental air samples (Alpha Results)

Figure 2b. Estimated anthropogenic alpha and beta activity on environmental air samples (Beta Results)

Figure 3. Comparison of algorithm calculated activity to the values expected using the arrangement in Figure 1. Here, the unused air filters are represented by the abscissa and the used air filters are represented by the ordinate. 


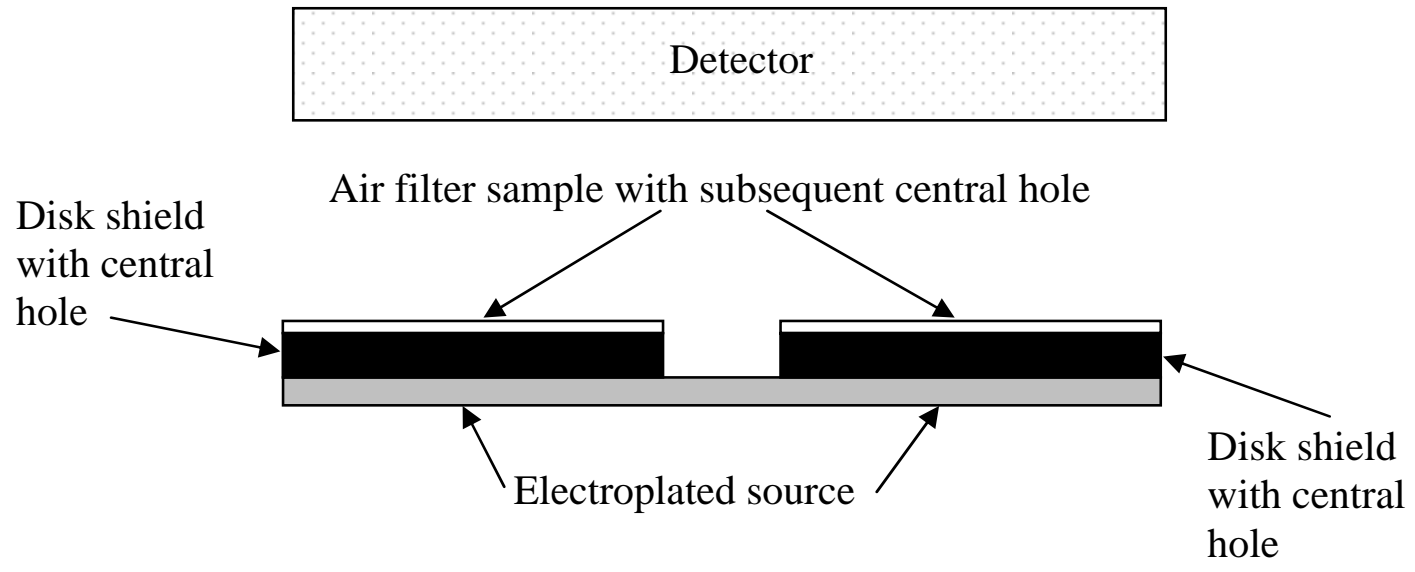

Figure 1. 


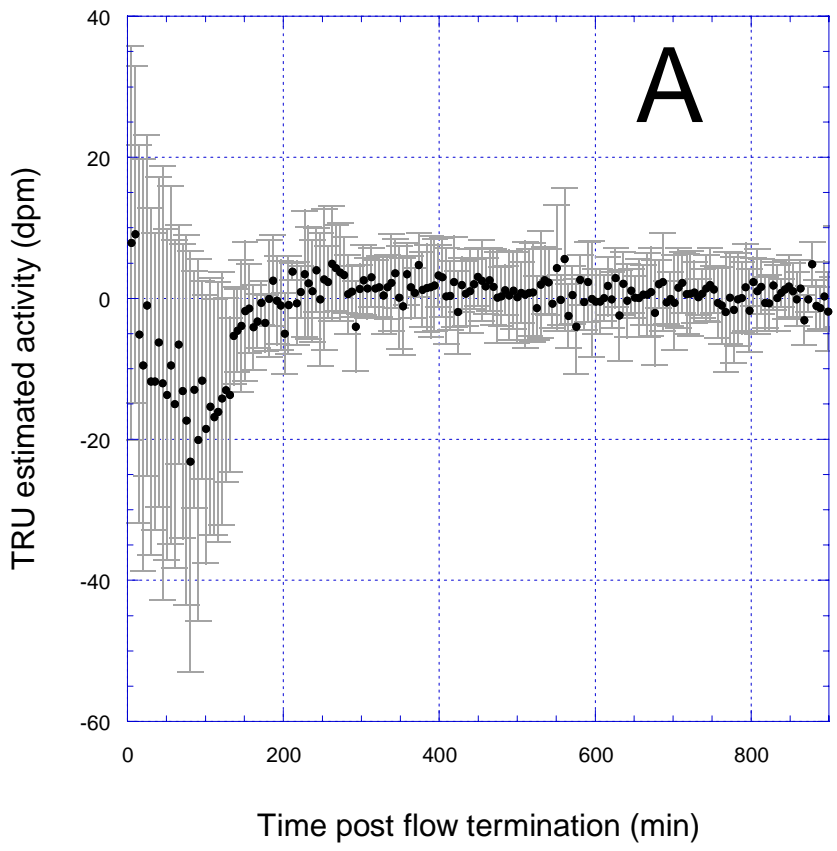

Figure 2a. 


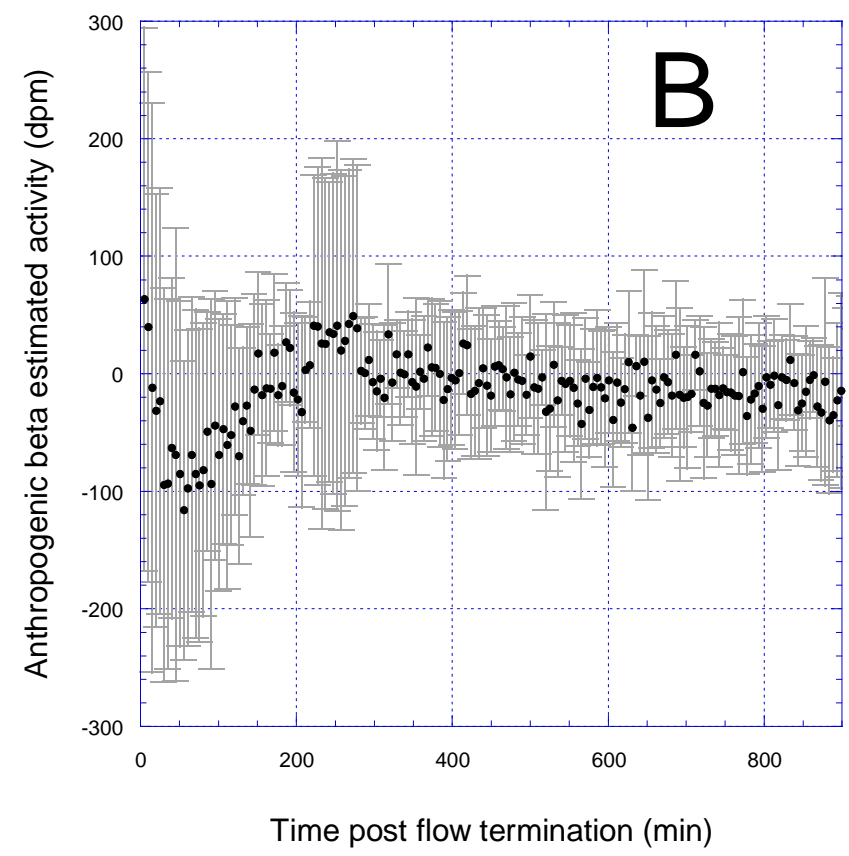

Figure 2b 


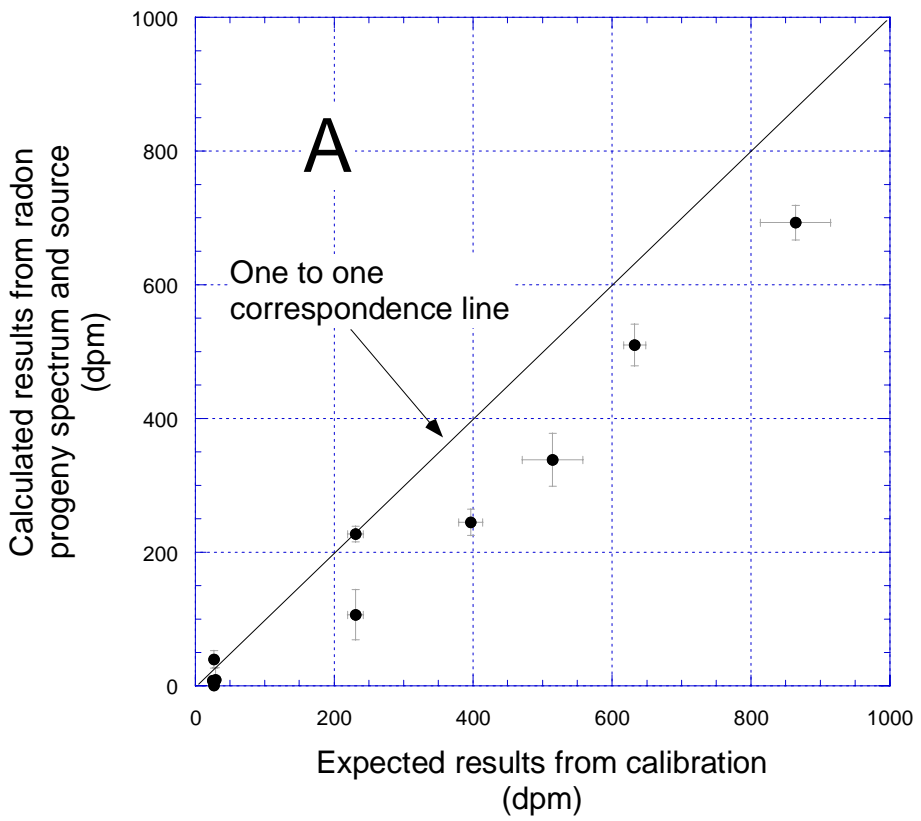

Figure 3. 